AperTO - Archivio Istituzionale Open Access dell'Università di Torino

\title{
Management of adrenal cancer: a 2013 update.
}

\section{This is the author's manuscript}

Original Citation:

Availability:

This version is available http://hdl.handle.net/2318/149674

since 2020-06-18T12:13:41Z

Published version:

DOI:10.1007/s40618-013-0049-2

Terms of use:

Open Access

Anyone can freely access the full text of works made available as "Open Access". Works made available under a Creative Commons license can be used according to the terms and conditions of said license. Use of all other works requires consent of the right holder (author or publisher) if not exempted from copyright protection by the applicable law. 


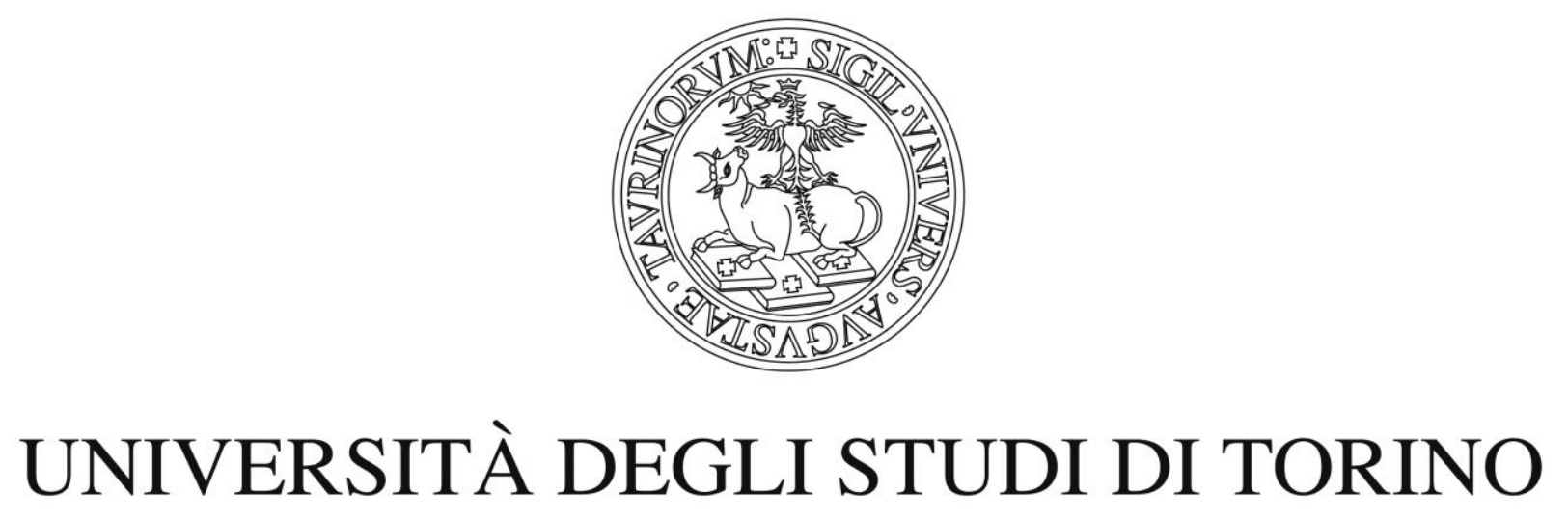

This is an author version of the contribution published on: Questa è la versione dell'autore dell'opera:

\section{MANAGEMENT OF ADRENAL CANCER: A 2013 UPDATE}

J Endocrinol Invest. Vol.37(3):207-17; 2014 Mar; DOI: 10.1007/s40618-013-0049-2

The definitive version is available at:

La versione definitiva è disponibile alla URL:

http://link.springer.com/article/10.1007\%2Fs40618-013-0049-2 


\section{MANAGEMENT OF ADRENAL CANCER: A 2013 UPDATE.}

\section{Terzolo ${ }^{1}$ MD, F. Daffara ${ }^{1}$ MD, A. Ardito ${ }^{1}$ MD, B. Zaggia ${ }^{1}$ MD, V. Basile ${ }^{1}$}

MD, L. Ferrari ${ }^{2}$ MD, A. Berruti ${ }^{2}$ MD.

${ }^{1}$ Internal Medicine I, Department of Clinical and Biological Sciences, University of Turin, and ${ }^{2}$ Medical Oncology, Department of Medical and Surgical Specialties, Radiological Sciences and Public Health, University of Brescia, Italy.

Running title: Management of ACC

Key words: Adjuvant treatment, adrenal cancer, adrenocortical carcinoma, chemotherapy, cisplatin, mitotane, overall survival, recurrence-free survival.

Address correspondence to:

M. Terzolo, MD

Internal Medicine I

Department of Clinical and Biological Sciences, University of Turin

San Luigi Gonzaga Hospital

Regione Gonzole, 10

10043 Orbassano, ITALY

Tel: ++ 390119026788

Fax: ++ 390116705456

e-mail: terzolo@usa.net

Financial disclosure: M.Terzolo received research grants and advisory honoraria by HRA Pharma. 


\begin{abstract}
Adrenocortical carcinoma (ACC) is a devastating tumor for either patients or their families because of short life expectancy and severe impact on quality of life. Due to the rarity of ACC, with a reported annual incidence of $0.5-2$ cases per million population, progress in the development of treatment options beyond surgery has been limited. Up to now, no personalized approach of ACC therapy has emerged, apart from plasma level - guided mitotane therapy, and no simple targetable molecular event has been identified from preclinical studies. Complete surgical removal of ACC is the only potentially curative approach and has the most important impact on patient's prognosis. Despite the limits of the available evidence, adjuvant mitotane therapy is currently recommended in many expert centers whenever the patients presents an elevated risk of recurrence. The management of patients with recurrent and metastatic disease is challenging and the prognosis if often poor. Mitotane monotherapy is indicated in the management of patients with a low tumor burden and/or more indolent disease while patients whose disease show an aggressive behavior need cytotoxic chemotherapy. The treatment of patients with advanced ACC may include loco-regional approaches such as surgery and radiofrequency ablation in addition to systemic therapies. The present review provides an updated overview of the management of ACC patients following surgery and of the management of ACC patients with advanced disease.
\end{abstract}




\section{INTRODUCTION}

Adrenocortical carcinoma (ACC) is a devastating tumor for either patients or their families because of short life expectancy and severe impact on quality of life, that is severely affected by metastatic progression, associated endocrine syndromes and treatment-related side effects. The fact that ACC has a first peak of incidence in young children and frequently occurs in women of childbearing age makes the management of this aggressive tumor even more challenging. In addition, currently available treatments of advanced ACC have limited efficacy and relevant toxicity that concurs to reduce quality of life (1-3).

Due to the rarity of ACC, with a reported annual incidence of $0.5-2$ cases per million population $(4,5)$, progress in the development of treatment options beyond surgery has been limited. Up to now, no personalized approach of ACC therapy has emerged, apart from plasma level - guided mitotane therapy, and no simple targetable molecular event has been identified from preclinical studies. Although generally regarded as one of the most aggressive endocrine tumors, ACC may present with a heterogeneous biological behavior ranging from almost indolent to extremely rapidly progressing tumors. Therefore, prognostic stratification of patients is of the utmost importance to tailor the treatment plan accordingly. However, we have limited knowledge of pathological and clinical criteria for risk stratification of ACC patients and the identification of prognostic and predictive biomarkers has to be actively pursued.

Management of ACC patients is challenging and demanding because physicians have to deal with either oncological issues, concerning tumor progression and metastatic development, or endocrinological issues, related to tumor secretion or specific treatment (mitotane effects on the endocrine system). Treatment of ACC is multi-modal, including surgery (often repeated), mitotane, cytotoxic agents, interventional radiology procedures, radiotherapy, and should be delivered by a multidisciplinary team within centers with adequate facilities (1-3, 6-8).

The objectives of this review are: i) to provide an updated overview of the management of ACC patients following surgery; ii) to provide an updated overview of the management of ACC patients with advanced disease. It has to be pointed out that the recommendations of this review are generally based on a low level of evidence and may represent the personal view of the authors. 


\section{MANAGEMENT OF ACC PATIENTS FOLLOWING SURGERY}

\section{Background}

Complete surgical removal of ACC is the only potentially curative approach and has the most important impact on patient's prognosis (1-3). Whether surgery should be done as open adrenalectomy or whether laparoscopic adrenalectomy may be safely performed in selected patients is matter of an ongoing debate (9-12). Despite this controversy, what is most important is that ACC surgery should be performed by experienced surgeons in referral centers with a high operation volume (7). A common finding of all surgical series is that recurrence after apparently complete resection is frequent and may be found in up to $70-85 \%$ of patients with high proliferation index or locally advanced disease (1-3, 8, 13-16). This observation makes a strong case for post-operative adjuvant treatment in ACC management.

In early years, many investigators considered the use of post-operative adjuvant therapy due to the high recurrence rate of ACC. The adrenolytic drug mitotane, an analogue of the insecticide dichlorodiphenyltrichloroethane (DDT) widely used for advanced ACC since the sixties, was considered the reference drug also for adjuvant treatment. However, those studies provided conflicting results for a number of reasons, namely small patient numbers, lack of control groups, unclear specification of treatment outcome (17). Given the lack of convincing evidence of the benefit of adjuvant mitotane, enthusiasm for this approach declined and no recommendation on post-operative adjunctive measures was released at the Ann Arbor Consensus Conference on ACC in 2003 (13).

In 2007, we published the results of a multicentric, retrospective analysis of post-operative management of 177 patients with ACC, who were recruited either at some centers in Italy where adjuvant mitotane was a standard following radical surgery or at other Italian centers where radical surgery was not followed by mitotane treatment. An independent cohort from Germany of patients who were not offered any post-operative treatment was also included. Recurrence-free survival (RFS) was significantly longer in the 47 patients treated with adjuvant mitotane, being 42 months versus 10 months and 25 months, respectively, in the two groups of 55 and 75 patients who were left untreated after surgery (18). Multivariate analysis confirmed that mitotane had an independent advantageous effect on RFS. The effect on overall survival (OS) was less apparent but nevertheless significant after adjusting for an imbalance of prognostic factors among the different groups (18). An important finding of the study is the acceptable adverse event rate, a finding that was likely due to the use of low doses of mitotane (1-5 g per day). Conversely, employment of high-dose mitotane in previous studies has been invariably accompanied by severe and disabling toxicity (17). 
Strengths of our study are a large patient cohort, availability of well-matched contemporary control groups, and predefined treatment allocation independent from patient's characteristics. Given its retrospective nature, however, this study cannot provide a high level of evidence in favor of adjuvant mitotane treatment. The publication of that study renewed interest on the use of mitotane in an adjuvant post-operative setting but raised also a fierce controversy based on the methodological flaws of that study (19).

Other adjunctive measures following surgical removal of ACC have received less attention. Cytotoxic agents have been rarely used in an adjuvant setting. The most interesting study is the one by Khan et al (20), who reported that 17 patients treated with the association of streptozotocin and mitotane had a significantly better RFS than 11 patients who did not receive any treatment after surgery. However, the study does not allow to discriminate the relative contribution of each drug. Despite the historical view that ACC is a radio-resistant tumor, the adjuvant use of radiotherapy has been explored in recent years. The argument remains conflicting, since two initial studies showed a significant reduction of local recurrences with radiation therapy, although the impact on RSF and OS was not fully clear $(21,22)$, while a more recent study did not show any benefit of adjuvant radiotherapy (23). Methodological differences among studies and the inevitable limits inherent to their retrospective nature may offer a reasonable explanation of this discrepancy.

In recent years, the adjuvant use of mitotane has become increasingly popular and in current practice the management of an ACC patient operated on certainly includes the option of giving mitotane. Therefore, the present review will focus on the use of mitotane as an adjuvant measure.

\section{Selection of patients to adjuvant mitotane}

Despite the limits of the available evidence, adjuvant mitotane therapy is currently recommended in many expert centers whenever the patients presents an elevated risk of recurrence. Differences do exist in the criteria used to define a high-risk condition, as exemplified in a recent position of an international panel of experts who agreed on stage I-II, complete (R0) resection and ki-67 $\leq 10 \%$ as markers of good prognosis, but a consensus was not found on stage III R0 ACC (24). In patients with good prognostic markers, the decision on adjuvant mitotane therapy may be individualized, whereas adjuvant mitotane is mandatory in the high-risk category (24). Following the ENS@T ACC staging system, stage III applies to locally invasive tumors characterized by infiltration in surrounding tissue, positive regional lymph nodes or a neoplastic thrombus in the vena cava or vena renalis (25). It is biologically plausible that tumor spread in regional lymph nodes or in the vein system may portend to a higher risk of recurrence than local infiltration and it is our opinion that subgroups at different risk of recurrence do exist among stage III ACC. Infrequently, a stage IV 
ACC, defined by presence of distant metastases (25), may be completely resected and has to be considered at a high risk of recurrence. The lowest risk applies to stage I and II ACC, being tumors localized in the adrenal gland with a with a size of $\leq 5 \mathrm{~cm}$ or $>5 \mathrm{~cm}$, respectively (25).

Recent data suggest that the proliferation activity of the tumor is the most important factor predicting risk of recurrence following R0 surgery. Assessment of the proliferation index Ki-67 is currently used to assess proliferation, despite some problems to harmonize immunohistochemical readings among different pathologists. In an European multicentric study, a threshold value at $10 \%$ was found to separate patients at good or worse prognosis with a hazard ratio of recurrence of 1.042 per each \% increase (26). Although the results of this study have still to be considered as preliminary, the availability of a large patient cohort totaling more than 500 patients represents a solid database to confirm the view that tumor proliferation is a strong determinant of patient survival. The value of ACC proliferation has been already appreciated in smaller series by the use of mitosis count $(27,28)$, that is likely the single most predictive factor of Weiss score. Conversely, Weiss score as a whole does not clearly indicate the probability of tumor recurrence $(28,29)$.

Resection status is another established adverse risk factor, being Rx (unknown), R1 (microscopically positive margins) and R2 (macroscopically positive margins) associated with progressively reduced RFS irrespectively of other risk factors (2-8).

A number of molecular markers, like matrix metalloproteinase type 2 (30), glucose transporter GLUT1 (31), SF1 (32), BUB1B and PINK1 (33) might potentially emerge in the future as powerful outcome predictors, but none of them has yet found a place in current management of ACC.

\section{Management of patients on adjuvant mitotane treatment}

No results from randomized controlled trial on adjuvant mitotane treatment are available. We have recently launched the first prospective randomized study -the ADIUVO trial (NCT00777244)which is currently recruiting at different European and North-American centers. The aim of this trial, whose results are not expected before 2016, is to compare the results of adjuvant mitotane with a strategy of no treatment on RFS (the study primary endpoint) in patients at low-intermediate risk of recurrence, defined by stage I to III ACC, R0 and Ki-67 $\leq 10 \%$ (www.adiuvo-trial.org). At our center, we are currently recommending low-risk patients to enter the trial while the remainders are offered adjuvant mitotane (Figure 1). Most patients seen in our practice actually belong to the highrisk category and are candidate to mitotane, which is an off-label prescription in adjuvant setting.

Whenever the decision of giving mitotane is taken, monitoring of plasma mitotane levels is currently considered a standard in the management of ACC patients treated adjuvantly because mitotane is known to have a narrow therapeutic window $(1-3,6,17)$. The concept that plasma levels 
of mitotane matter for both therapeutic efficacy and drug-related toxicity has been developed in the clinical scenario of advanced ACC (34-36). Mitotane concentration should be kept above $14 \mathrm{mg} / \mathrm{l}$ to achieve the most from treatment (36). The same target of $14 \mathrm{mg} / \mathrm{l}$ has been pursued by many experts also when using mitotane as an adjuvant measure, despite the lack of specific data in disease-free patients $(1-3,17)$. Results of a very recent retrospective analysis provided evidence that this strategy is sound also in the adjuvant setting (37). Of 122 ACC patients who were radically resected, 63 patients reached and maintained target mitotane concentrations during follow-up. They had a significantly lower rate of recurrence than 59 patients who failed to keep mitotane levels as high. Mitotane was an independent factor influencing RFS at a multivariate analysis while the prolongation of OS did not reach levels of statistical significance (37). This may be due to the relatively short follow-up duration and should not downplay the effect of mitotane. The study actually provided a further, although indirect, evidence in favor to adjuvant mitotane.

What is the best dosing regimen to initiate mitotane therapy is currently debated and either highdose or low-dose regimens are employed. A high-dose regimen has the merit of inducing a faster rise of plasma mitotane that may translate in a more rapid action of the drug $(38,39)$. It is concerning that several weeks of treatment are needed to attain blood drug levels conferring therapeutic efficacy $(1-3,17,40)$, since this may cause a window of under-treatment following surgery during which tumor remnants may grow and progress. With a low dose regimen, a longer time needed to achieve therapeutic concentrations may be anticipated. However, RFS did not differ between patients attaining target mitotane concentrations within 3 months and patients who did not in a retrospective study of 122 patients who underwent a monitored mitotane treatment (37). It is likely that a continuous maintenance of target mitotane concentrations during follow-up be of more importance that a faster attainment of desired levels. Furthermore, a high-dose regimen requires an intensive follow-up and may be more frequently associated with side effects, while a low dose regimen is better tolerated and easier to manage in an outpatient setting $(1,17)$.

In our practice, we start mitotane treatment as soon as possible after surgery with 2 tablets (1 gram) daily. The patient is instructed to add one tablet every 4-7 days, depending on drug tolerability, targeting a dose of 6-8 g daily, or the maximum tolerated dose. We try to accommodate mitotane schedule to patient's tolerance with the aim of increasing compliance with treatment and minimizing the impact on patient's quality of life. However, we are strongly committed to target serum mitotane concentrations of 14-20 mg/l. Monitoring of mitotane concentrations is done on a regular basis, after 4 to 6 weeks from treatment start and thereafter every 4-8 weeks until target levels are reached to adjust dosage accordingly. Then, mitotane dose can be usually reduced and 
monitoring is done less frequently. We manage mitotane toxicity by reducing the daily dose to the previously tolerated one, or discontinuing treatment for a while allowing resolution of complaints.

Mitotane monitoring is key for the management of patients treated adjuvantly to guide dose adjustments with the aim of targeting mitotane concentrations of therapeutic relevance while avoiding severe toxicity. Experience with mitotane before availability of drug monitoring was afflicted by severe and disabling toxicity (15). In Europe, mitotane monitoring is readily available as a free service provided by the company distributing mitotane (info@lysodren-europe.com). Thus, this is no longer a barrier to optimal practice.

Mitotane is a toxic drug and unwanted effects are observed in almost all patients, but toxicity is usually mild and tolerable, if managed properly, with a low-dose schedule. Most commonly, patients complain of gastrointestinal manifestations, such as nausea and diarrhea, early in the course of treatment. These unwanted effects occur independently on mitotane levels. They can be managed with temporary dose reduction, or delay of dose increments, and symptomatic therapy $(1,17)$.

Clinically significant liver toxicity is characterized by a marked increase in transaminases and bilirubin, but is infrequently observed although predisposing conditions are present. Conversely, elevation in GGT levels is an universal finding without any clinical impact unless values are exceedingly elevated. Central neurologic toxicity is more closely associated with mitotane concentrations $>20 \mathrm{mg} / \mathrm{l}$ but subtler symptoms, such as memory impairment or attention deficit, may be observed in some patients at lower concentrations $(1,17)$. A great individual variability in the susceptibility to mitotane-related unwanted effects is apparent for causes that are still unknown $(1-3,17,40)$. Recent data from our group suggest that individual response to mitotane, in terms of circulating drug levels, is at least partially genetically determined (41). Evaluation of gene polymorphisms involved in mitotane metabolism, like CYP2B6 and ABCB1 genes, may allow predicting which patients may be more responsive and/or more prone to unwanted effects.

Because of the adrenolytic effect of mitotane, all patients should receive glucocorticoid replacement to prevent adrenal insufficiency. Hydrocortisone (Cortisone acetate) is used at doses that are almost double than in Addison's disease, due to an enhanced metabolic clearance rate of glucocorticoids induced by mitotane (1-3). Mitotane enhances cytochrome P450 3A4 enzyme activity resulting in rapid inactivation of more than $50 \%$ of administered hydrocortisone (42). An inadequate treatment of adrenal insufficiency increases mitotane-related toxicity, particularly gastrointestinal side-effects, and reduces tolerance. Adequacy of replacement has to be judged mostly on clinical grounds and routine biochemical tests; hormone monitoring is of limited utility (17). Mineralocorticoid supplementation is not mandatory in all patients because mitotane has only a limited effect on the zona glomerulosa. Frank elevation of PRA heralds mineracolorticoid 
insufficiency $(17,40)$. In mitotane-treated patients, a derangement in thyroid function characterized by low FT4 levels without a compensatory rise in TSH is frequently observed early in the course of treatment. This finding usually prompts thyroxin replacement (40). In vitro evidence that mitotane is able to inhibit TSH secretion at the pituitary level, thus explaining a hormone pattern alike central hypothyroidism, has been published (43).

Gonadal function is also compromised during mitotane treatment by mechanisms that are still to be completely elucidated. Most women maintain regular cycles and may even become pregnant on treatment. However, some women develop ovarian cysts and olygomenorrhea with possible vaginal bleedings. In treated men, sexual dysfunction is more common due to inhibition of testosterone and DHT synthesis $(40,42)$. Gynecomastia is also frequently seen and may be partially due to the weak estrogen-like activity of mitotane (40). Sex steroid replacement may become necessary to treat erectile dysfunction in some patients but may worsen gynecomastia. However, the best way to replace gonadal steroids remains disputed (42).

Patients should be carefully informed of the goals of treatment, including the importance to target mitotane concentrations of $14-20 \mathrm{mg} / \mathrm{l}$, to motivate them to cope with unwanted effects and to remain compliant with a cumbersome poly-pharmacologic regimen. To this aim, it is important the establishment of a close patient-physician relationship to induce and maintain adherence to treatment. Patients may seek advice frequently and their local physicians are usually unfamiliar with mitotane; thus, it is necessary to give timely counseling to keep patients on treatment.

There are insufficient data to define what is the optimal duration of adjuvant mitotane treatment. In our practice, we are currently recommending longer periods of treatment, at least in patients showing a good compliance, the minimal duration of treatment being 2 years. Since most ACC recurrences occur within 5 years from surgery, this period is considered as a landmark to consider discontinuation of treatment. 


\section{MANAGEMENT OF ACC PATIENTS WITH ADVANCED DISEASE}

\section{Background}

The management of patients with recurrent and metastatic disease is challenging and the prognosis if often poor. However, a minority of patients with metastatic disease may show a rather indolent disease course. Several prognostic factors such as time since diagnosis, presence of hepatic or bone metastases, number of metastatic lesions and number of tumoral organs involved, high mitotic rate (20 per 50 high-power field), and atypical mitoses in the primary tumor have been found to predict survival in patients with metastatic ACC $(44,45)$. Two previous reports identified cortisol secretion as a negative prognostic factor in metastatic ACC patients. In a large singleinstitution French series including 202 patients with different disease stages, cortisol excess was found to be an independent prognostic factor for OS and was predictive of subsequent metastatic disease in the subset of patients with stage I to III (46). Similar results were obtained from a series of 72 Italian patients with metastatic ACC submitted to chemotherapy with EDP (Etoposide, Doxorubicin and Cisplatin) plus mitotane (47).

The treatment of advanced/metastatic patients include loco-regional approaches such as surgery and radiofrequency ablation (RFA) in addition to systemic therapies. In presence of isolated locoregional recurrence or oligo-metastatic disease, surgery can lead to improved survival (14), so an aggressive surgical approach to achieve complete resection (R0) may be advisable. Conversely, tumor debulking offers little benefit and may be considered in patients with functional tumors not controlled by medical treatment.

In patients who are not candidates for surgery, percutaneous image-guided RFA is a locally effective treatment. RFA was well tolerated in a small series of 15 ACC recurrences, with 53\% of patients demonstrating decrease in tumor size or loss of enhancement on imaging (48). RFA in combination with surgical resection may allow better disease control in the setting of olygometastatic disease.

\section{Chemotherapy and mitotane in the management of metastatic ACC}

Systemic treatments in the management of patients with metastatic disease include mitotane alone or mitotane in combination with chemotherapy. Single agent mitotane has a modest activity and response rates between $13 \%$ and $31 \%$ have been reported (Table 1). Most of the responses are of limited duration, and complete responses rarely occur. Monitoring mitotane serum levels is mandatory since it was demonstrated that disease responses are mainly confined in patients 
attaining and maintaining over time serum levels within the therapeutic range $(14-20 \mathrm{mg} / \mathrm{l})(34,35)$. Mitotane serum levels within the therapeutic range are also predictive of prolonged survival (36). Beside of its antitumor effect, mitotane is a strong inhibitor of adrenal steroidogenesis and it has a compelling indication in patients with endocrine symptoms, although the rate of success in controlling hormone excess is not well known $(2,3)$. Owing to the latency of mitotane to attain the therapeutic range, mitotane monotherapy is indicated in the management of patients with a low tumor burden and/or more indolent disease. For patients whose disease show an aggressive behavior, cytotoxic chemotherapy is required (Figure 2).

Whether cytotoxic chemotherapy is effective or not in the management of ACC was a matter of debate for a long time. The results of several small studies have demonstrated that chemotherapy administered alone has a limited activity and the combination of chemotherapy with mitotane seems to increase the response rate (Table 2). Mitotane may have a synergistic effect on chemotherapy activity thanks to the ability to reverse multidrug resistance mediated by P-glycoprotein expression (49). ACC produces high levels of the multidrug resistance protein MDR1 (also known as Pglycoprotein) which functions as an ATP-dependent drug efflux pump, transporting out of the cell hydrophobic cytotoxic agents such as doxorubicin, vinblastine, and paclitaxel. However, the effect of mitotane on multidrug resistance has been questioned (50).

The efficacy of chemotherapy was demonstrated by the results of a large prospective multicenter multinational phase III study (FIRM-ACT) that compared the efficacy of 2 chemotherapy regimens: Etoposide, Doxorubicin, Cisplatin, and Mitotane (EDP-M) and Streptozotocin and Mitotane (Sz-M) (51). Three hundred and four patients were prospectively enrolled in about 6 years. Patients with disease progression to the first-line treatment received the alternate regimen. EDP-M was superior to Sz-M both in terms of disease response rate and progression free survival (PFS). Analysis of OS also favored patients initially randomized to receive EDP-M but the difference just failed to attain statistical significance. The explorative analysis performed in the subset of patients that crossed to the alternative regimen upon progression showed a significant improvement of PFS and OS in patients receiving EDP-M in second line as opposed to those receiving Sz-M. Therefore, the efficacy of EDP plus mitotane as second-line therapy attenuated its advantage as first-line therapy and affected the OS results. These limitation notwithstanding, it should be noted that the 5-year survival increased from about $7 \%$ in patients initially randomized to receive Sz-M to about $15 \%$ in patients receiving EDP-M. On the basis of the results of this trial, the European Society for Medical Oncology guidelines recommend EDP-M as the first-line therapy for ACC requiring cytotoxic therapy (6). The EDP-M regimen however is quite toxic and the combination of cisplatin 
plus mitotane may constitute a reasonable alternative for patients that appears to be not fit for EDPM.

The efficacy of chemotherapy plus mitotane is overall modest but highly variable among patients. At our center, we treated with chemotherapy plus mitotane (mainly EDP-M) 180 metastatic ACC patients over 20 years, and 4 of them (2.2\%) obtained an extremely favorable outcome. All of them had a PFS greater than 5 years and only one had a disease progression after 6.5 years, the remaining patients are still alive and disease free after 11, 5 and 6 years, respectively. Two patients attained a complete response and the remainders attained a partial response and then became disease free after surgical removal of the residual disease (52). These data suggests that cytotoxic therapy plus mitotane could potentially cure few metastatic ACC patients. These data undeline the importance to identifying factors that may predict chemotherapy efficacy in order to select patients for this aggressive strategy and avoid unhelpful toxicity in patients not destined to obtain any benefit.

The results of second-line therapy in patients with disease progression to platinum containing regimens plus mitotane were as a whole modest and in most studies disappointing (see the following chapter).

Our group has developed a metronomic schedule of cytotoxic drugs in order to overcome drug resistance and limit patient toxicity. Metronomic chemotherapy is the administration of cytotoxic drugs at low doses, on a frequent or continuous schedule, with no extended interruption. This approach can target tumor cells indirectly, since it can affect the endothelium of the growing tumor vasculature and stimulates anticancer immune response. One multicenter Italian study assessed the activity of the combination of gemcitabine administered on day 1 and 8 in association with fluoropyrimidines, such as 5-fluorouracil and capecitabine, administered on a metronomic schedule (53). Since the main goal of metronomic approach is to induce tumor dormancy, both tumor response and disease stabilization (the so called clinical benefit) were considered. Among the 28 patients who entered the study, 13 (46.4\%) obtained a clinical benefit lasting 4 months at least. Two patients attained a tumor response according to RECIST criteria, one of them complete. The patient who attained a complete response remained free from progression for 48 months, then tumor progressed and the patient died 6 months later. These data suggest that this combination regimen may have some efficacy, at least in a subset of patients. Since all patients had progressive ACC to platinum containing regimens plus mitotane, gemcitabine plus capecitabine could be potentially considered non-cross resistant to cisplatin-containing regimens. Despite all patients included in the trial had disease progression on mitotane, the drug was not interrupted. Patients who had serum mitotane above the therapeutic threshold had a better time to progression than those who did not. 
These data suggest that the synergism between mitotane and chemotherapy can persist when further chemotherapeutic agents are introduced. This hypothesis deserve further study because practice varies as to continuing mitotane after progression to this treatment.

Metronomic chemotherapy may be occasionally active also when administered after several treatment lines. We recently described two cases with metastatic ACC with a rather long disease history that attained a durable disease response with oral cyclophosphamide and oral etoposide, respectively, both administered on a metronomic schedule (54). These data suggest that metronomic chemotherapy may be active in patients bearing a rather indolent disease.

\section{Molecular target agents in the management of metastatic ACC}

Several oncogenes, growth factors and tumor suppressor genes have been implicated in ACC tumorigenesis. The most important genomic alterations involve the p53 system, the Insulin Growth Factor Receptor (IGFR) and the Wnt/B-catenin signalling pathway. In addition, also the Epidermal Growth Factor Receptor (EGR) and neoangiogenesis are considered potential targets.

As outlined in Table 3, several small phase II trials have tested the efficacy of molecular agents targeting EGFR, angiogenesis, IGFR, and mTOR pathways. These treatments were administered in pre-treated patients. The molecular agents employed were tested alone, in combination with chemotherapy, or with other molecular target agents. As a whole, these trials obtained poor results. EGFR target agents (gefitinib and erlotinib) either administered alone, or in combination with gemcitabine, were not active $(55,56)$. Three trials tested anti-angioenetic drugs, two of them testing the association of bevacizumab with capecitabine (57) and sorafenib with weekly paclitaxel (58), respectively, led to negative results. In a multicenter prospective phase II German trial, sunitinib obtained disease stabilization lasting more than 4 months in 5 patients out of the 36 consecutively enrolled (13.8\%); 4 of them showed a modest increase of ACC lesions less than $20 \%$ and therefore below the threshold for progression according to the RECIST criteria and only one a decrease of tumor burden (below the threshold of response) (59).

The reasons why these trials were substantially negative are not clear and several hypotheses can be raised. First, poor patients selection, because of i) inclusion of heavily pretreated cases that may have led to emerging of multi-resistant tumor clones, or ii) inclusion of tumor that do not express the molecular target. As an example, EFR mutations in ACC are extremely rare and this may have accounted for the poor response obtained with the EGFR inhibitors in unselected ACC patients. It has been shown that these drugs are active in the small subset of patients whose non small cell lung cancers harbor EGFR mutation (60). Second, the patients included in molecular target therapy trials were pretreated with mitotane, a drug with a very long half life (more than 40 days) and whose 
biological activity usually persists for months after drug withdrawal. Many small molecular target agents are substrates of the p450 dependent enzyme CYP3A4 that is induced by mitotane (61). Therefore, pretreatment with mitotane may have reduced the antitumor efficacy of these agents.

Better results were obtained by figitumumab, a monoclonal antibody against IGFR-1, either administered alone (62) or in combination with the mTOR inhibitor temsirolimus (63, 64). As shown in Table 3, durable disease stabilizations were obtained in a relevant proportion of patients. In our opinion, figitumumab deserves to be further explored in the management of advanced ACC. Being a monoclonal antibody, this drug is not metabolized by the CYP3A4 enzyme and its pharmacokinetic may be not negatively influenced by mitotane. A prospective phase II trial testing the activity of figitumumab in association with mitotane in ACC relapsed/refractory or primary not removable by surgery is currently recruiting in USA (Trial No NCT00778817).

The most important study in this arena was a multicenter, multinational, prospective, randomized, placebo-controlled clinical trial recently conducted aimed to test the efficacy of OSI 906-301, a small molecule IGFR inhibitor, as second- /third-line approach in advanced/metastatic ACC patients (Trial No: NCT00924989). This trial has completed patient accrual in June 2011 and the final results are expected in 2014. 


\section{LEGENDS}

Figure 1. Management strategies following surgical extirpation of ACC.

Figure 2. Management strategies of patients with advanced ACC. 